\title{
Impulsivity and impairment in cognitive functions in criminals
}

\begin{abstract}
Impulsivity is related with some of the abnormal behaviors like anti-social acts, violence and crime. It is suggested that impulsivity is associated with malfunctions in cognitive functions especially abnormality in frontal lobe of brain. Cognitive functions are a wide range of processes such as attention, attention switching, maintenance of concentration, control and interference-control, space and time integrity, regulation, working memory, logic thinking and problem solving. In risky behaviors like crime and law-breaking a risky decision and impulsivity are seen that formed by a combination of impairment in cognitive function. In this mini review, impulsivity as a personality trait is introduced and its relationship with decision making and cognitive functions is explained.
\end{abstract}

Keywords: criminal psychology, impulsivity, cognitive functions, criminal
Volume 5 Issue I - 2017

\author{
Elham Foroozandeh \\ Ph.D. in Psychology, Department of Psychology, Islamic Azad \\ University, Iran
}

Correspondence: Elham Foroozandeh, Ph.D. in Psychology, Department of Psychology, Islamic Azad University, Naein Branch, Naein, Iran, Tel 98 9133004695,

Email Elham_for@yahoo.com

Received: April 29, 2017| Published: June 21, 2017

\section{Introduction}

Impulsivity is related with some of the psychological distresses in human community, including anti-social behaviors, violence and crime. Recent surveys have shown that impulsivity is one of the main risk factors in personality structure for crime. Impulse behavior is defined as urges and cravings to perform an action in response to a real external or subjective stimulus. Impulsivity is a wide range of behaviors that are less thinking about them, are immature with seeking a reward or pleasure goal, have high risks and in the core of them can be seen the significant social unintended consequences. These behaviors are shown with inability to resist and avoid an impulse, drive or temptation to perform a harmful behavior for person or the others. Before impulsive behavior, person usually feels increasingly tension and arousal and sometimes stress whereas pleasure is mixed with theses emotions. Conducting and completing the impulsive behavior would result gratification and immediate relief but after doing it combination of regret, guilt, self-blame and fear may be experienced. One of the main features that cause impulsive behavior is self-destructive behavior like substance abuse and suicide attempts. ${ }^{1}$

It is anticipated that impulsive behaviors are associated with brain functions especially executive functions. Executive functions play key roles in guiding and managing behaviors. During developmental period, the child will be better in cognitive functions and mental tasks which are related to brain mechanisms, so the child will be able to perform more difficult and complicated tasks. ${ }^{2}$ Executive functions are including cognitive structure that explain behaviors which are linked to frontal lobe function. These functions are essential in all complex cognitive processes or new goal-oriented assignments. Executive functions are the ability to maintain appropriate posture for problem-solving and achieve a higher cognitive skills of the brain. Regarding the areas of executive functions, they are related to attention, logic thinking and problem solving skills and include activities such as attention switching, maintenance of concentration, control and interference-control, space and time integrity, regulation and working memory. In fact the executive function is the ability to process information. ${ }^{3}$
More complicated behavior especially social behavior requires more executive functions. ${ }^{4}$ Anti-social behaviors such as crime may be reflects of increasingly impairment of cognitive functions. The brain has two kinds of cognitive function. The first kind is the function based on the legal and thinking factors that are actions that regulate and control behaviors and generally known as executive functions. The other kind are unsystematic functions that their bases are emotions, feelings and desires. Executive functions and social recognition may change decisions in risky situations like crime. Some risky behaviors related to substance abuse are connected to impairment in pre-frontal cortex and hippocampus areas. ${ }^{5}$ It is shown that in persons with violent and criminal behavior, neuropsychiatric abnormalities have high rates so it is a suggestion that there is an association between aggressive dyscontrol and brain injury, especially involving the frontal lobes. ${ }^{6}$ In psychiatric patients although the location of the abnormal regions varied, they showed significantly lower relative brain glucose metabolic values in medial temporal and prefrontal cortices than did normal comparison subjects. These regions have been implicated as substrates for aggression and impulsivity, and their dysfunction may have contributed to the patients' violent behavior. ${ }^{7}$

Executive functions are cognitive processes that regulate attention capacity, abstract reasoning and integration of cognitive skill sets and help in achieving targets in the form of decisions, sustained attention, information organizing, planning and flexibility. Executive functions are very different components and from the perspective of cognitive neuropsychology they are associated with the wide network of the functions of the frontal cortex and contains a wide range of cognitive and meta-cognitive processes, such as self-regulation and social skills that develop in period of a childhood and adolescence period. Their expansion and growth like the other skills happen in childhood. According to these findings, it is suggested that impulse persons experience difficulty and disability in performing cognitive task successfully. This problem is more clear when an impulse person needs deep and permanent thinking to do tasks, in this situation he/ she use a careless strategy and weak way to solve problems. Barkley ${ }^{8}$ showed self-control as a personality trait and brain executive functions are overlap and behavioral inhibition is more essential component in 
interaction of these processes. Also it is reported that that an increase in sustained attention causes lowers impulsivity. ${ }^{9}$ So it can said that impulsivity is related to crime and law breaking behaviors an on the other hand it is associated with neuro-cognitive functions like attention and planning.

\section{Discussion}

Cognitive functions as the most important functions of frontal lobe in brain, have crucial role in doing complex cognitive task like decision making. Although it seems that the decision-making process is simple, but in fact it is one of the most complex and sometimes difficult issues that persons face because decision making is a choosing between competing values and the consequences of choosing a value is ambiguous and unpredictable. It can be said that the decision making process is a process that all available options should be studied one by one and in this process the options will be remove one after another. This trend will continue as long as an option remains and a final decision is made. This process needs a high ability in executive function. In the risky decision, a person is faced with the choice that has the profit and loss account in current time and in the future. However, these gains and losses associated with some degree of risk and uncertainty. Risky decisions is considered a decision with a positive short term, but negative long-term consequences, and is characterized as a disorder when a long-term goal is a sacrifice for an instant gratified goal. This is what is known as a key factor in many abnormal behaviors including aggressive behaviors, substance abuse, antisocial personality disorder, suicidal tendencies and sexual risky behaviors.

\section{Conclusion}

In conclusion it is suggested that to understand crime, it is helpful know more about brain functions specially neuro-cognitive processes and their relationship with impulsivity that is a basic factor in aggressive behaviors, anti-social traits and criminal behaviors.

\section{Acknowledgments}

None.

\section{Conflicts of interest}

None.

\section{References}

1. Zmani N, Vahid A, Moghanloo Ataee V, et al. Compare the effectiveness of two methods of dialectical behavior therapy and cognitive behavioral therapy to improve symptoms in patients with major depression with suicidal thoughts and impulsive behavior. Journal of Ilam University of Medical Sciences. 2013;22(5):45-54.

2. Alizadeh H. Relationship of neuro-cognitive executive functions with developmental disorders. New Ideas in Cognitive Science. 2008;8(4):5770 .

3. Voigt RG, Katusic SK, Colligan RC, et al. Academic Achievement in Adults with a History of Childhood Attention-Deficit/Hyperactivity Disorder: A Population-Based Prospective Study. J Dev Behav Pediatr. 2017;38(1):1-11.

4. Bahramkhani M, Darvishi N, Keshavarz Z, et al. Comparison of executive functions in children with autism and normal and their relationship with mathematics and reading ability. Quarterly Journal of Rehabilitation. 2013;13(5):128-135.

5. Bayrami M, Mhmdzadgan R, Movahedi Y, et al. Evaluation of cognitive performance in drug abusers, addicts treated with methadone and normal individuals. Journal of Substance Abuse and Addiction Studies. 2014;9(34):37-23.

6. Brower MC, Price B. Neuropsychiatry of frontal lobe dysfunction in violent and criminal behaviour: a critical review. J Neurol Neurosurg Psychiatry. 2001;71(6):720-726.

7. Volkow ND, Tancredi LR, Grant C, et al. Brain glucose metabolism in violent psychiatric patients: a preliminary study. Psychiatry Res. 1995;61(4):243-253.

8. Barkley R. Barkley deficits in executive functioning scale (BDEFS). Guilford Press, USA. 2011.

9. Logemann HN, Lansbergen MM, Van Os TW, et al. The effectiveness of EEG-feedback on attention, impulsivity and EEG: a sham feedback controlled study. Neurosci Lett. 2010;479(1):49-53. 